\title{
Estrategia didáctica para el desarrollo transversal de la competencia comunicativa en la formación profesional de los estudiantes de educación superior
}

\author{
Teaching strategy for development of tranversalcommunicative competence in vocational \\ training of higher education students
}

\section{Maciel Castaño Hernández}

Docente de la Institución Educativa Técnica de Turbana, Bolívar, docente -Tutor Programa Todos a Aprender, Ministerio de Educación. Especialista en Pedagogía de la Lengua y la Literatura, Licenciada en Lengua Castellana y Comunicación de la Universidad de Pamplona., Estudiante de maestría en educación.macielcastanoh@gmail.com

\section{Ana Luz Echenique Escandón}

Docente de la Institución Educativa Técnica Agropecuaria de Sincerin, docente-Tutor Programa Todos a Aprender, Ministerio de Educación. Licenciada en Trabajo Social de la Universidad de Cartagena. Estudiante de maestría en educación. anechees@hotmail.com

Para citar este artículo: Hernandez M., Echenique A. (2017). Estrategia didáctica para el desarrollo transversal de la competencia comunicativa en la formación profesional de los estudiantes de educación superior. Escenarios, 15(1), pp. 119-130. Doi: http:/ / dx.doi.org/10.15665/esc.v15i1.1125

Recibido: enero 10 de 2017

Aceptado: febrero 15 de 2017

\section{RESUMEN}

La educación colombiana en su deseo por orientar la formación de individuos integrales y competentes ante los distintos mercados laborales y sociales ha dispuesto el desarrollo de competencias genéricas o transversales, que son evaluadas por el ICFES, mediante el examen Saber Pro. La Universidad Autónoma del Caribe en coherencia con estas necesidades actuales, se esfuerza por que en sus programas se desarrollen transversalmente varias competencias, entre ellas la competencia comunicativa, objeto de la presente propuesta.

Por tal razón, se parte de un análisis del estado de esta competencia, tomando aleatoriamente una muestra de los resultados de la prueba Saber Pro 2014-2015, en el que se evidencia la necesidad de fortalecer la lectura crítica y competencia escritora, debido a los bajos resultados obtenidos en la misma.

Con base en esta necesidad, se orienta la investigación cuyo objetivo es proponer una estrategia de enseñanza aprendizaje para el desarrollo transversal de la competencia comunicativa lectora y escritora, en educación Superior. Partimos de la revisión bibliográfica de teorías existentes, así como otras investigaciones a nivel local, nacional e internacional cuyo interés ha sido la competencia comunicativa, específicamente: Lectura crítica y competencia escrita.

Palabras clave: Competencias comunicativas - Transversalidad -Lectura crítica -producción escrita. 


\begin{abstract}
Colombian education guides the formation of integral and competent people who will confront the variety of labour and social markets. So, it has provided the development of generic or transversal competences, which are evaluated by the ICFES, using the test: Saber Pro.

The Caribbean Autonomous University being coherent to the current needs, endeavors to develop, in a transversal way, different competences in their programs; among them the communicative one, which is the aim of this pedagogical proposal.

That's why we draw from the premise, that it's important the analysis of the condition of this competence. We took a random sample of the test Saber Pro in 2014-2015, in which we observed the need to strengthen the comprehensible reading and the writing competence due to the low results of the test.

Based on this need, our research aims to propose a teaching and learning strategy for the transversal development of reading and writing communicative competence in higher education. We based it on the literature review of existing theories related to the same topic, as well as other research works in a local, national and international field.
\end{abstract}

Keywords: communicative skills -- Transversality - critical Reading - written production.

\section{INTRODUCCIÓN}

Lograr niveles altos de lectura crítica y un buen desarrollo de la comunicación escrita de los estudiantes de educación superior, ha sido un tema complejo y se podría decir un gran desafío en el proceso de enseñanza de las mismas; estas competencias son evaluadas por el ICFES como competencias genéricas, las cuales son consideradas transversales a todas las áreas del conocimiento ( Icfes, Saber Pro, 2014).

En referencia a lo anterior, según estudios a nivel nacional e internacional de diferentes universidades y análisis suministrados por el ICFES entre estos, Saber en Breve, 2015, los resultados obtenidos por estudiantes de distintas facultades en universidades oficiales, tienden a quedar en un nivel regular, demostrando una falencia en la competencia comunicativa, aún cuando en su pensum académico contemplan módulos relacionados con el desarrollo de estas competencia.

En relación con lo anterior, la Universidad Autónoma Del Caribe, en el logro de sus propó- sitos educativos plantea la organización de un currículo que parta del análisis de los contextos formativos, académicos y laborales actuales para lograr que los programas involucren el desarrollo de competencias en coherencia con las expectativas y necesidades del mercado laboral a nivel nacional, regional y local, dirigiendo con especial interés procesos hacia el fortalecimiento o mejora del desarrollo de la competencia comunicativa, específicamente en comprensión y evaluación de textos científicos y disciplinares.

Para la estructuración del currículo esta Universidad, en los lineamientos curriculares, ha determinado tres tipos de competencias profesionales según el área de formación curricular, denominadas: competencias básicas institucionales, competencias básicas de la profesión y competencias específicas de la profesión. Las primeras se relacionan con las competencias requeridas para todos los profesionales independientemente del campo disciplinar; el segundo tipo se refiere a competencias comunes a un grupo de profesiones afines y las terceras constituyen competencias definidas concretamente para un profesional determinado. 
Establece seis competencias básicas institucionales (Uso de las TIC, procesamiento de la información y pensamiento lógico, sostenibilidad, juicio moral y competencia comunicativa) consideradas transversales, en cuanto deben ser abordadas en todas las disciplinas, durante el desarrollo de la vida universitaria.

\section{¿Qué dice la norma?}

En Colombia desde la ley 115 (Ley General de Educación), el Plan Decenal de Educación y el sistema de Evaluación del ICFES, se establecen una serie de normas y / o lineamientos para el desarrollo de las competencias comunicativas en el proceso educativo;en los artículos asignados para cada nivel de educación, dispone el desarrollo de las competencias comunicativas como elementos que fortalecen la convivencia, la adquisición de valores y el desarrollo de habilidades lectoras y escritoras como: la comprensión de textos, la capacidad crítica y propositiva. En este sentido dispone los siguientes artículos: Artículo 16 ( para educación preescolar, Artículo 20 y 21 de Educación Básica, literal b) y literal c), Artículo 22, literal a), Artículo 30. de Educación media, literal g)

Desde el 2009, el ICFES, con la evaluación ECAES introduce una prueba de Comprensión Lectora, siguiendo la conceptualización de la competencia comunicativa en tres dimensiones: interpretar, argumentar, proponer; y distinguiendo tres niveles de lectura. A partir del 2011, de la prueba de comprensión lectora se pasa a la prueba de Lectura Crítica de SABER PRO. (ICFES,2013).

Cabe mencionar que el tema de las competencias comunicativa y su transversalidad en el currículo ha preocupado en gran medida, a instituciones de nivel superior de educación, a razón de esto se resalta a nivel internacional, el siguiente artículo: "Alfabetización académica y competencia Comunicativa en educación superior" (2013) de Juan Antonio Núñez Cortés doctor de la Universidad Autónoma de Madrid,quienen el artículo plantea que "El desempeño en la lectura y la escritura se erige como una competencia clave para el aprendizaje en la educación superior. Así, el desarrollo de la competencia comunicativa en la universidad del siglo XXI tiene que favorecer que los estudiantes universitarios, protagonistas de un nuevo paradigma de enseñanza-aprendizaje, conjuguen información, conocimiento y producción del mismo"(AHELO, 2012).

\section{MÉTODO}

El presente proyecto desarrolla una metodología mixta teniendo en cuenta que sigue unas etapas, las cuales permiten sentar las bases para el planteamiento de estrategias pedagógicas que aporten al desarrollo transversal de la competencia comunicativa. Estas consisten en:

-En realizar un análisis de los resultados de la prueba Saber pro en lo referente a las competencias transversales, en este caso: Lectura crítica y competencia escritora, atendiendo a lo dispuesto por el ICFES.

- Identificación y descripción del problema, a partir del análisis cuantitativo de los resultados Saber Pro (Lectura crítica y competencia escritora) en una muestra de estudiantes de la facultad Administración de Empresas.

- Revisión del marco conceptual y teorías de distintos autores sobre competencia comunicativa y procesos transversales en el currículo, así como la comparación entre las distintas teorías haciendo planteamientos críticos que permitan proponer otras estrategias que direccionen la transversalidad de la competencia comunicativa en la formación profesional de los estudiantes de educación Superior, mirando que la razón primordial de su desarrollo es formar personas competentes no solo en el espacio académico si no para su contexto personal y social.

- Finalmente se espera diseñar una estrategia cuyo propósito será fortalecer el desarrollo de la competencia comunicativa en los estudiantes de educación superior. 


\section{Población}

Para el desarrollo de la investigación se toma como población los estudiantes de los cuatro primeros semestres del programa de Administración de Empresas en modalidad presencial de la universidad Autónoma del Caribe de Barranquilla. La muestra seleccionada es de 172 estudiantes. matriculados.

\section{DISCUSIÓN Y REFLEXIÓN}

El presente trabajo aborda la competencia comunicativa, resaltando además su importancia en el desarrollo personal y social del individuo, desde todos los contextos en los que este se desenvuelva. Considerando que:

"Desarrollar los procesos de comprensión, producción y socialización discursiva es una labor minuciosa, ardua y delicada, esto debido a que se debe tener en cuenta no solo la competencia lingüística (dimensiones pragmático, semánti$\mathrm{CO}$, fonológico- ortográfico y gramatical), sino también los aspectos estratégicos y socioculturales en los que surgirán dichos textos. (ARNAO, M, INGRID, M, 2014)

La prueba ECAES, 2009, introdujo la Comprensión Lectora, siguiendo la conceptualización de la competencia comunicativa en tres dimensiones: interpretar, argumentar, proponer; y distinguiendo tres niveles de lectura (literal, inferencial y crítico intertextual).

En este sentido Cassany, (2003) se refiere al primer nivel como: la "lectura de línea o comprensión literal de las palabras que componen el fragmento, a la capacidad de decodificar su significado semántico: elegir la acepción adecuada al contexto, de entre las que incluye el diccionario, y obtener todos los semas pertinentes". El segundo nivel o inferencial requiere una lectura "entre líneas, se refiere a la capacidad de recuperar los implícitos convocados en el texto, que contribuyen de manera decisiva a elaborar su coherencia global y a construir el significado relevante del escrito". El tercer nivel de lectura, crítico intertextual requiere leer detrás de las líneas, capacidad de comprender qué pretende conseguir el autor, por qué lo escribió, con qué otros discursos se relaciona (contexto, comunidad, etc.); y a poder articular una opinión personal respecto a las ideas que expone, con argumentos coincidentes o no. p.4.

Las competencias evaluadas por el ICFES motivan el presente estudio, considerado pertinente, dados los resultados obtenidos por los estudiantes en un test diagnóstico realizado por la universidad y también en los resultados de las Pruebas Saber Pro. El test consistió en mirar el estado de desarrollo de las competencias transversales, este fue aplicado a 150 estudiantes de los cuatro primeros semestres de la UAC, consistió en preguntas de comprensión lectora, que evaluaban el nivel literal, inferencial y crítico de la lectura, cuyos resultados se describen a continuación:

- En orden descendente: El 79\% de los evaluados manejan un nivel bueno de comprensión literal, el $48 \%$ mostró nivel regular de comprensión inferencial, el $44 \%$ un nivel satisfactorio de comprensión crítica. Como se aprecia en la medida que incrementa el nivel de comprensión lectora son más bajos los resultados en cantidad y calidad en esta.

Esta investigación realiza entonces, un análisis a los resultados en las pruebas Saber Pro 20142015 obtenidos por 172 estudiantes de la facultad de Administración de Empresas de la UAC, observando un déficit en la lectura crítica y competencia escritora. La gráfica $N^{\circ} 1$, muestra un comparativo de los resultados de los estudiantes, los cuales son clasificados en Quintiles, siendo el quintil I el más bajo y el quintil V, el más alto. 


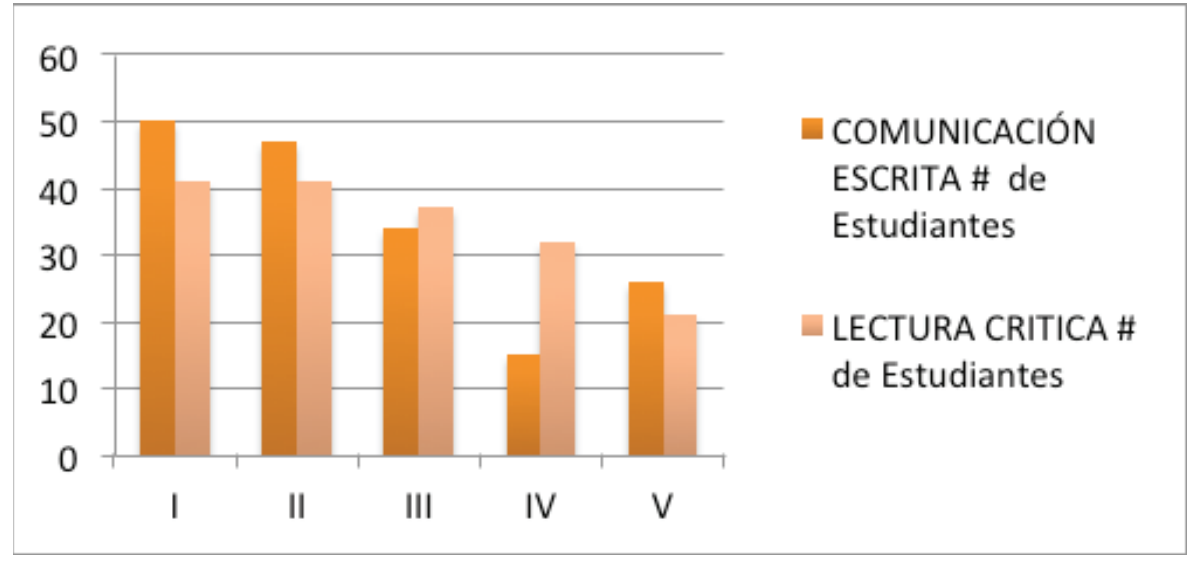

\begin{tabular}{|c|c|c|}
\hline Quintil & $\begin{array}{c}\text { COMUNICACION } \\
\text { Escruta } \\
\text { \# de } \\
\text { Estudiantes }\end{array}$ & $\begin{array}{c}\text { ECTurA } \\
\text { Cermca } \\
\text { \# de } \\
\text { Estudiantes }\end{array}$ \\
\hline I & 50 & 41 \\
\hline II & 47 & 41 \\
\hline III & 34 & 37 \\
\hline IV & 15 & 32 \\
\hline$V$ & 26 & 21 \\
\hline
\end{tabular}
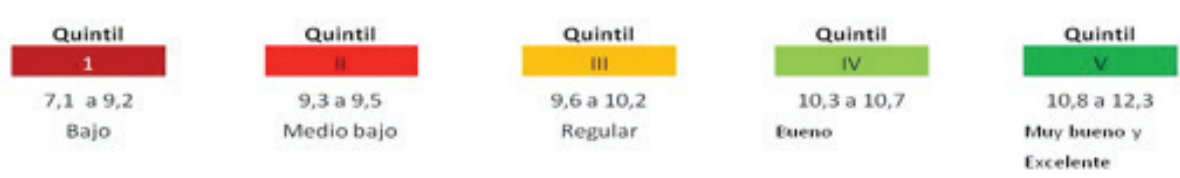

Gráfica $N^{\circ} 1$

Según la gráfica se concluye que: En la competencia escritora 50 estudiantes se ubican en los niveles, bajo, y 47 en el nivel medio bajo, lo cual quiere decir que: el estudiante ofreció un esbozo de respuesta, sin embargo, esta carece de comunicación asertiva, ideas concatenadas y de un texto organizado.

Treinta y cuatro (34) estudiantes se ubican en el nivel regular, y bueno, esto indica: elaboran textos mejor desarrollados, en los que afloran elementos sintácticos, argumentos y proposiciones satisfactoriamente utilizadas. En los niveles superiores. 15 de los estudiantes se distribuyen en los niveles muy bueno y 26 estudiantes se ubican en excelente, quiere decir que los textos producidos son coherentes, con una conexión explícita entre las distintas secciones, en los que hay rastros de una planeación previa y que, al desarrollar la intención comunicativa, tienen en cuenta al interlocutor.

En el componente de lectura crítica el mayor número de los estudiantes evaluados se ubica entre los niveles bajo y medio bajo y un menor número en el nivel regular, logrando solo:

Identificar y entender los contenidos locales que conforman un texto. Esto es capacidad de identificar y comprender los eventos, ideas, afirmaciones y demás elementos locales del texto. Comprensión del significado de palabras, expresiones o frases que aparecen explícitamente en el mismo. Cabe anotar que un estudiante que no cumpla con los requerimientos de este nivel mínimo, no podrá avanzar a los siguientes. De los 172 estudiantes evaluados, 41 se ubican en el nivel bajo y 41 en el nivel muy bajo, queriendo decir que no alcanzan este nivel. Seguidamente 32 estudiantes se ubican en el nivel bueno, 37 se ubica en el nivel Mínimo, cuya habilidad evalúa: Comprender cómo se articulan las partes de un texto para darle un sentido global. Esta competencia consiste en la capacidad de comprender cómo se relacionan semántica y formalmente los elementos locales que constituyen un texto, de manera que éste adquiera un sentido global. Finalmente 21 estudiantes se ubican en los niveles muy bueno y excelente. Consiste en: Reflexionar a partir de un texto y evaluar su contenido. Esta competencia consiste en la capacidad de enfrentar el texto críticamente. Incluye evaluar la validez de argumentos, advertir supuestos, derivar implicaciones, reconocer estrategias argumentativas y retóricas, relacionar los contenidos con variables contextuales, etc. Esta es la competencia propiamente crítica, para su desarrollo requiere las dos anteriores. 
Estos dos aspectos llevan a la construcción de la problemática de este proyecto, la cual se sintetiza en la siguiente pregunta de investigación: ¿Qué estrategia de enseñanza aprendizaje contribuiría a la transversalidad de la competencia comunicativa, específicamente lectura crítica y competencia escritora de los estudiantes de educación superior de la Universidad Autónoma del Caribe?

\section{Competencia comunicativa e importancia en el contexto universitario y social.}

\subsection{Conceptualización de competencia}

El diseño del presente proyecto como ya es dicho, tiene como finalidad realizar un análisis del nivel de desarrollo de la competencia comunicativa en estudiantes de educación Superior, específicamente lo relacionado a la lectura crítica y competencia escritora, consideradas transversales a todas las áreas del conocimiento, puesto que son el paso para lograr la adquisición y producción de conocimiento, el desarrollo de la vida social y laboral del individuo. A razón de lo anterior se definen los siguientes conceptos, los cuales orientan el estudio de la competencia comunicativa.

Competencia: "Conjunto de conocimientos, habilidades, actitudes, comprensiones y disposiciones cognitivas, metacognitivas, socioafectivas, comunicativas y psicomotoras apropiadamente relacionadas entre sí para facilitar el desempeño flexible, eficaz y con sentido de una actividad o de cierto tipo de tareas en contextos relativamente nuevos y retadores". El ICFES considera tres tipos de competencias: interpretativa, argumentativa y propositiva. ICFES (2013).

El siguiente esquema presenta un comparativo entre la concepción inicial de competencia comunicativa, planteada por Chomsky, quien se refiere a la competencia lingüística y Hyme, que introduce el término competencia comunicativa:
Esquema $\mathrm{N}^{\circ}: 1$ La competencia comunicativa

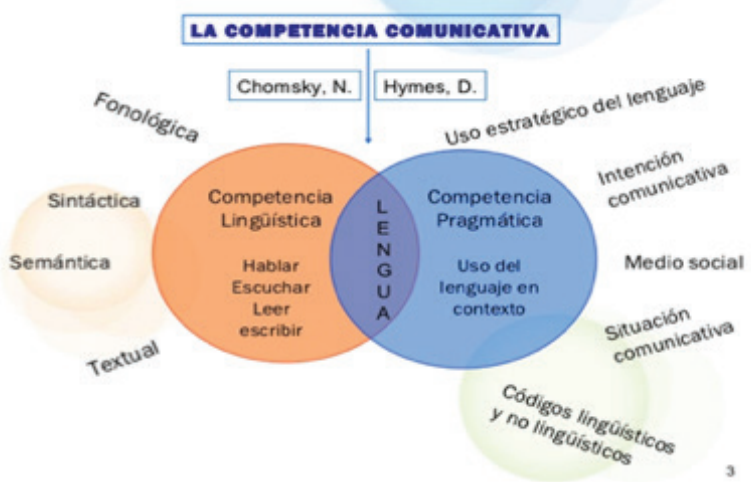

RINCÓN, C. La competencia Comunicativa. Unidad 11.

La lengua como sistema de signos corresponde al dominio semiótico, y su función esencial es significar. La lengua en funcionamiento, en uso, corresponde al dominio semántico, y su función básica es comunicar.

"La competencia comunicativa comprende las aptitudes y los conocimientos que un individuo debe tener para poder utilizar sistemas lingüísticos y translingüísticos que están a su disposición para comunicarse como miembro de una comunidad sociocultural dada". (GIRON y VALLEJO, 1.992).

En palabras de Bubnova, la parte lingüística refleja la parte pasiva del lenguaje y es el nivel semiótico de la comunicación, pero lo translingüístico es determinante en la comprensión activa del mensaje y representa la parte efectiva de la comunicación (Bubnova, 2006).

Por su parte Niño, (citado por: Lily Bermúdez y Liliana González, 2011) confirma que la competencia comunicativa es saber comunicarse en un campo del conocimiento y un saber aplicarlo; saberes que comprenden conocimientos, habilidades, actitudes y valores (precondiciones, criterios, usos, reglas y normas) para realizar actos comunicativos eficientes en un contexto determinado, según necesidades y propósitos. 
Subcompetencias de la competencia comunicativa

La competencia comunicativa requiere el desarrollo de varias subcompetencias: Dell Hymes( EEUU, 1972) atribuye al concepto de competencia comunicativa, la competencia lingüística (Chomsky), la competencia socio-lingüística, la competencia discursiva y la competencia estratégica. Apuntando hacia el aspecto verbal y pragmático, pero dejando de lado el proceso de producción de significados. Bachman, Barcelona, Gedisa, (1990/1995) nos habla de "habilidad lingüística comunicativa" que puede ser descrita como compuesta del conocimiento, o competencia, y la capacidad para poner en práctica o ejecutar esa competencia en un uso de la lengua adecuado y contextualizado. Canale(1995, Madrid, España): Hace la siguiente discriminación de las competencias: Gramatical, sociolingüisica, discursiva y estratégica, competencia gramatical (se refiere al dominio del conocimiento lingüístico). La competencia sociolingüística (relacionada con la adecuación de las producciones al contexto. competencia discursiva (se refiere a los modos en que se combinan unidades gramaticales para formar textos, hablados o escritos, coherentes y completos). Competencia estratégica (relacionada con el dominio de estrategias de comunicación verbal y no verbal que pueden suplir carencias y lograr una comunicación efectiva). Aportando elementos importantes como son la competencia discursiva y la competencia estratégica). Segùn Mauricio Pilleux, Chile, 2001, agrega la competencia psicolingüística, entendiéndose como una "disciplina encargada de descubrir cómo se produce y se comprende el lenguaje por un lado y cómo se adquiere y se pierde el lenguaje por otro (Frias,2002)

En el presente trabajo se considera importante el fortalecimiento de la competencia comunicativa, como elemento transversal en todos los niveles educativos, trazando como de suma importancia lo planteado por Canale(1995, Madrid, España) en cuanto al desarrollo de otras sub competencias como es la gramatical, la sociolingüistica, la discursiva y estratégica, agregando además la competencia pragmática, a la cual se refiere Bachman y Mauricio Pilleux, dado que el dominio de la competencia comunicativa requiere:

Producir y comprender mensajes, ideas, opiniones, orales o escritas, de manera eficaz, eficiente y pertinente según el contexto y acto de habla del que se encuentre participando, en situaciones reales de comunicación, usando o desarrollando habilidades de tipo gramaticales, textuales, discursivas, entre otros y que le permiten transmitir de manera clara un mensaje.

Entre otras concepciones la competencia comunicativa se refiere a producir y comprender mensajes, ideas, opiniones, orales o escritas, de manera eficaz, eficiente y pertinente según el contexto y acto de habla del que se encuentre participando, en situaciones reales de comunicación, usando o desarrollando habilidades de tipo gramaticales, textuales, discursivas, entre otros y que le permiten transmitir de manera clara un mensaje. Peña. 2008.

En esta línea queremos fundamentar la presente propuesta, dirigiéndose a potenciar el desarrollo y transversalidad de la competencia comunicativa en el individuo como parte importante en el desarrollo integral de este, teniendo en cuenta que involucra habilidades presentes tanto en la vida cotidiana, como en su desempeño laboral como: hablar, leer, escribir, escuchar.

\section{Competencias Transversales o Genéricas}

El Icfes y la universidad promueven el desarrollo transversal de la competencia comunicativa , pues por medio de ella es posible la adquisición del conocimiento, la producción del mismo y la relación social con el mundo. Según el Diccionario de la Real Academia de la Lengua Española, se define la palabra transversal como aquello que se halla o se extiende atravesado de un lado a otro.

Por otra parte transversalidad es un concepto que surge con las reformas educativas para "atravesar el currículum" desde una dimensión transdisciplinar que cruza a todos los compo- 
nentes del mismo, acentuando la dimensión procedimental, actitudinal y axiológica del componente educativo. PANTOJA. 2007.

A partir de lo anterior el concepto transversalidad inicialmente era entendido como aquellos tópicos no contemplados en el plan curricular, pero que dado su importancia para el desarrollo social y axiológico del ser humano eran asumidos mediante planes o programas ajustados según los requerimientos o fines de cada área.

De esta forma según Pantoja el aprendizaje tomaba un carácter holístico en tanto que le permite al estudiante una mayor perspectiva de su formación, respondiendo a las exigencias sociales, y laborales. Por lo que se vuelve más significativo dado que relaciona y utiliza lo aprendido en su contexto y vida social. El aprendizaje también toma un enfoque constructivista desarrollando habilidades investigativas sistemáticas, organizativas y de producción de información.

Consecuentemente, La transversalidad desde su inicio ha sido una forma distinta de entender la enseñanza, un compromiso social del profesorado de colaboración entre la escuela y comunidad, en la búsqueda y propuesta de soluciones a los problemas que cotidianamente nos afectan. La visión globalizadora que ofrecían las transversales es una opción de la que no se puede renunciar y es tarea que debe mantener el profesorado al elaborar sus Proyectos Educativos ( Gavidia-Catalán, Aguilar, V y Carratalá R. 2011)

En acuerdo con la anterior teoría es de gran importancia que durante la educación universitaria, se continúe el desarrollo de las compe- tencias comunicativas (Hablar, leer, escribir, escuchar), desde la complejidad de este nivel, de modo que el proceso de formación de los estudiantes sea secuencial, progresivo e integral. Considerando también lo reglamentado en nuestro sistema educativo colombiano, el cual dispone el desarrollo de las competencias genéricas o transversales, entre estas la comunicativa, enfocándose fundamentalmente en la Lectura crítica y competencia escrita, como procesos fundamentales que permiten a la persona la interpretación del mundo, de la simultaneidad de contextos, culturas y tipologías textuales que hoy abundan, desde una óptica crítica y propositiva.

\section{$\mathrm{Al}$ respecto}

Cassany, (2003) La persona crítica es la que: Comprende autónomamente el propósito lingüístico, las intenciones pragmáticas y los puntos de vista particulares que subyacen a los discursos que le rodean; • toma conciencia del contexto (contenido cognitivo, género discursivo, comunidad de hablantes, etc.) desde el que se han elaborado dichos discursos; - puede construir discursos alternativos, que defiendan sus posiciones personales y que se vinculan polifónicamente o intertextualmente con los anteriores; • utiliza todos los recursos lingüísticos disponibles para conseguir representar discursivamente sus opiniones a través de esos segundos discursos." p,2.

El examen Saber Pro realiza una clasificación de las competencias que abarcan la lectura crítica y la competencia escritora: 
Cuadro 1: VARIABLES ANALIZADAS

\begin{tabular}{|c|l|l|}
\hline $\begin{array}{c}\text { COMPETENCIAS } \\
\text { TRANSVERSALES }\end{array}$ & COMPONENTES & \multicolumn{1}{c|}{ COMPETENCIA } \\
\hline \multirow{3}{*}{ LECTURA CRÍTICA } & SINTÁCTICO & $\begin{array}{l}\text { Comprender cómo se articulan las partes de un Texto para } \\
\text { darle un sentido global. }\end{array}$ \\
\cline { 2 - 3 } & SEMÁNTICO & $\begin{array}{l}\text { Identificar y entender los contenidos locales que conforman } \\
\text { un texto. }\end{array}$ \\
\cline { 2 - 3 } & PRAGMÁTICO & $\begin{array}{l}\text { Reflexionar a partir de un texto y evaluar } \\
\text { su contenido. }\end{array}$ \\
\hline \multirow{3}{*}{ PRODUCCIÓN ESCRITA } & SINTÁCTICO & $\begin{array}{l}\text { La organización del texto. (Planeación y jerarquización del } \\
\text { texto) }\end{array}$ \\
\cline { 2 - 3 } & SEMÁNTICO & $\begin{array}{l}\text { El planteamiento que se hace en el texto. (Propiedades del } \\
\text { texto: adecuación, coherencia, cohesión) }\end{array}$ \\
\cline { 2 - 3 } & PRAGMÁTICO & $\begin{array}{l}\text { La forma de la expresión: Elección del lenguaje según inter- } \\
\text { locutor e intención comunicativa. }\end{array}$ \\
\hline
\end{tabular}

ICFES, Saber Pro. Módulo de comunicación Escrita, (2015)

Por otra parte, también es importante tener en cuenta algunos aspectos como la atención, la percepción y el pensamiento a la hora de enfrentar la lectura o escritura de un texto (ROQUE, 2010), las cuales se definen a continuación, partiendo del aporte que hacen en el proceso de comprensión textual:

ATENCIÓN: Puede definirse como un proceso de selección de información o como un recurso energético a distribuir $n$ las diferentes operaciones, según se trate de recibir información o de ejecutar tareas. Cuando se recepciona información, ejemplo: durante la lectura o cuando se realiza una tarea, ejemplo: transcripción de un texto. La atención se puede dar en tres niveles o aspectos: Atentividad, atención selectiva, concentración

PERCEPCIÓN: La percepción es el proceso cognitivo que permite:

a. La interpretación de la información, o capacidad que tiene el individuo de encontrar significado a los estímulos que se enfrenta.

b. El reconocimiento de patrones. O elementos guardados en la memoria (conocimiento previo) los cuales son utilizados al momento de enfrentarse a situaciones o estímulos. En nuestro caso: La lectura o escritura. (ROQUE,2010)

PENSAMIENTO: El pensamiento permite el manejo, transformación y uso funcional del conocimiento que se aprende, para superar las dificultades que se encuentran en el entorno. Pensar implica un conjunto de habilidades (Beltran; 1998), que permite interactuar mentalmente y en el plano de las ideas. Algunas habilidades que permiten aprender conocimientos, transformarlos y utilizarlos son: observar, identificar, establecer secuencias, comparar, clasificar, identificar relaciones causa-efecto, inferir información de textos y analizar. (ROQUE,2010)

De otra parte cabe mencionar que el tema de las competencias comunicativa y su transversalidad en el currículo ha preocupado a instituciones de nivel superior de educación, a razón de esto se resalta a nivel internacional:"Alfabetización académica y competencia Comunicativa en educación superior" (2013) Juan Antonio Núñez Cortés doctor de la Universidad Autónoma de Madrid en el artículo plantea que "El desempeño en la lectura y la escritura se erige como una competencia clave para el aprendizaje en la educación superior. 
Así, el desarrollo de la competencia comunicativa en la universidad del siglo XXI tiene que favorecer que los estudiantes universitarios, protagonistas de un nuevo paradigma de enseñanza-aprendizaje, conjuguen información, conocimiento y producción del mismo" (AHELO, 2012).

Por otra parte la Universidad de Zulia plantea la necesidad de cambiar el currículo rígido por uno que permita el desarrollo de procesos e innovación de la práctica educativa, un tipo de enseñanza que involucre la transversalidad curricular y por ende la integralidad. (TORRES, A. FERNANDEZ E., 2014). "muestra un análisis de la percepción de los estudiantes de Educación Superior sobre el desarrollo de su competencia comunicativa al entrar y al salir de la universidad.

A nivel nacional la UNISANTANDER, cuya investigación "Las competencias transversales en el currículo de las unidades tecnológicas de Santander" conceptualiza las competencias transversales y su importancia de ser involucradas en el plan de estudios, resaltando como competencias básicas transversales: Comunicación de ideas, comprensión y análisis de textos, comprensión lectora, entre otras. (OCAZIONEZ, 2007)

\section{La pontificia Universidad Javeriana:}

PÉREZ, M. y RINCON, G (2013), apoyado en el discurso de Paula Carlino y Emilia Ferreiro, manifiesta que la lectura y escritura se debe abordar desde el espacio escolar al profesional, el cual es más específico, está marcado por constantes retos relacionados con los tipos de textos con los que no hemos tenido experiencia pre- via. Entonces en la formación profesional universitaria es menester que los estudiantes se enfrenten con diversos géneros (por ejemplo, el tecnológico, el científico y el jurídico), que contienen textos organizados en modos predominantemente expositivos y argumentativos, con temáticas especializadas, la mayoría de gran complejidad, que requieren mayores grados de abstracción."

En el mismo sentido la Universidad Autónoma del Caribe presenta dos módulos: El primero constituye en una "Estrategia para la enseñanza-aprendizaje activo en la Universidad", cuyo propósito es que el docente de la UNIAUTONOMA, reconozca aspectos influyentes en la comprensión lectora, y el segundo: "La comprensión lectora como Estrategia de enseñanza -aprendizaje en la educación universitaria". El cual brinda estrategias para la evaluación de la comprensión lectora, la noción de estructura proposicional del texto y el reconocimiento de estructuras textuales, así como estrategias de elaboración, de organización y estrategias para la metacomprensión.

Aún cuando existen muchos estudios sobre el desarrollo de la competencia comunicativa, ésta por su carácter complejo, podríamos decir en constante desarrollo, es de imperiosa necesidad continuar su estudio, en búsqueda de estrategias que la potencialicen.

\section{PROPUESTA}

En vista que la presente investigación aún se encuentra en desarrollo, desde el análisis realizado a las teorías de distintos autores, la propuesta a formular se orientará según lo planteado en el siguiente esquema: 
Esquema 2: Premisas sobre el desarrollo transversal de la competencia comunicativa.

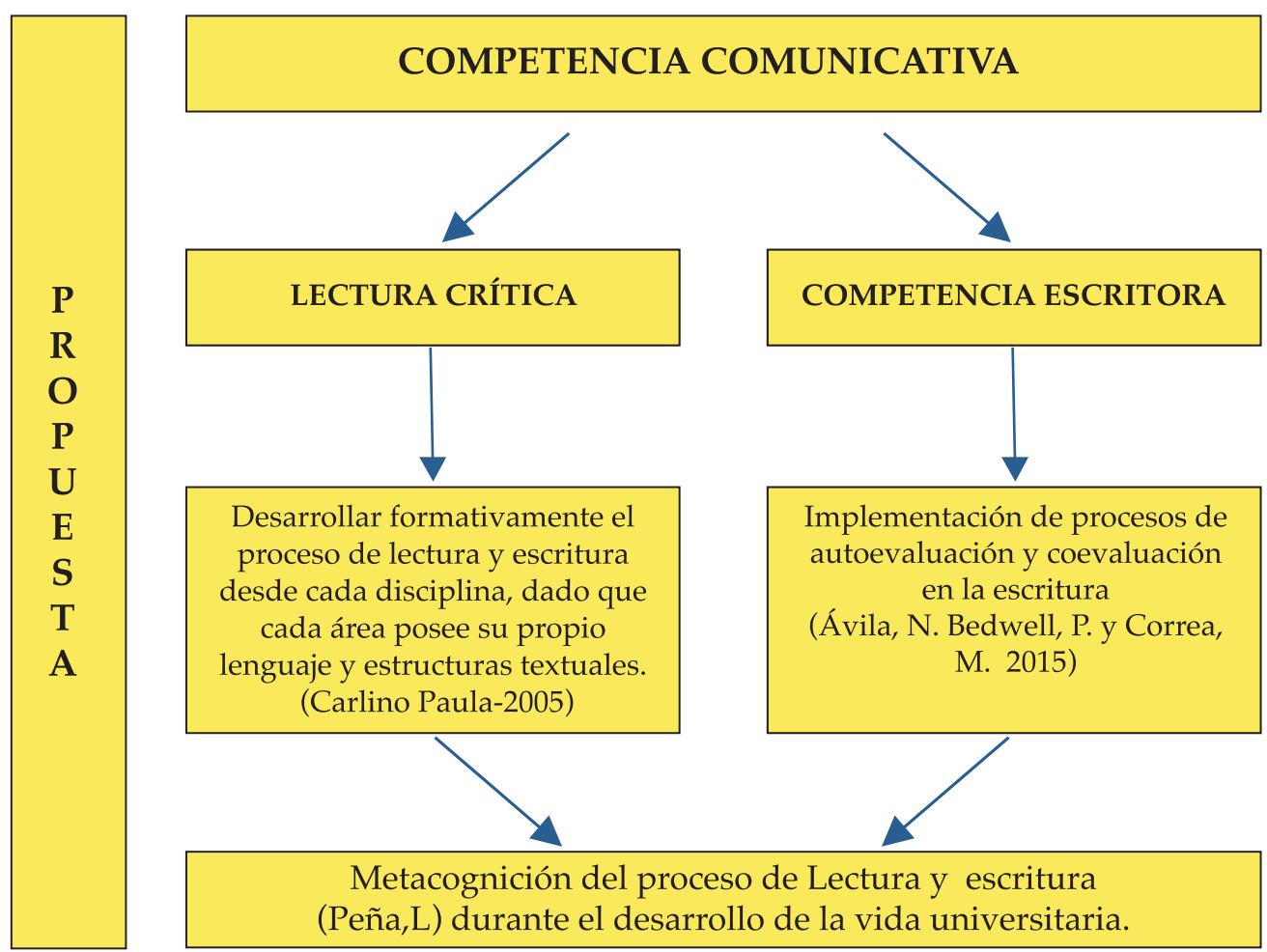

\section{CONCLUSIONES}

La presente investigación involucra primeramente un informe descriptivo de los resultados Saber Pro de una muestra de estudiantes de la UAC, en la competencia comunicativa: Lectura crítica y competencia escritora, dicho informe presenta bajos resultados en esta competencia, realidad que lleva a un análisis de teorías existentes sobre el desarrollo de la competencia comunicativa y el concepto de transversalidad en el currículo, además de estudios realizados por otras universidades a los cuales les ha interesado el tema, teniendo en cuenta que la competencia comunicativa es de carácter indispensable en la vida social y cotidiana del ser humano y que por ende se hace ineludible su desarrollo en la formación básica y profesional del individuo. (Ley 30, Educación superior).

Es por esto la necesidad de redireccionar los procesos de aprendizaje en la educación superior, de tal manera que esta competencia se evidencie y transversalice en la organización curricular, y que cada disciplina sea responsable de su desarrollo en la formación del estudiante universitario, (Carlino,P, 2010) favoreciendo así una mejor comprensión de los tipos y estructuras textuales, formas de expresión de cada área del saber, así como la comprensión de los mismos, Pues tanto la lectura como la escritura se consideran procesos indispensables en la adquisición y producción del conocimiento, y la comunicación social, en cuanto al desarrollo de la escritura, una estrategia a considerar sería la utilización de la autoevaluación y la coevalución del proceso de escritura en el aula (ÁVILA, N. BEDWELL, P. y CORREA, M. 2015), lo que permitiría a los estudiantes un constante análisis del texto escrito, de los discursos, los propósitos, las estructuras textuales y mecanismos de cohesión, a partir de los textos del otro y sobre el propio, despertando además la crítica y argumentación.

\section{REFERENCIAS BIBLIOGRÁFICAS}

ARNAO, M, INGRID, M, (2014). Percepción de los estudiantes de Educación superior sobre el desarrollo de su competencia comunicativa. Revista de investigación y cultura. Vol. 3, N 1. ÁVILA, N. BEDWELL, P. y CORREA, M. ( 2015) 
Rúbricas y otras herramientas para desarrollar la escritura en el aula. Santiago de Chile.

CARLINO, P. IGLESIA, P. Y LAXALT, I. (2010). Leer y escribir en la formación de profesores secundarios de diversas disciplinas: qué dicen los docentes que se hace. Jornadas Nacionales Cátedra UNESCO de Lectura y Escritura Lectura, escritura y aprendizaje disciplinar. Universidad Nacional de Río Cuarto, Río Cuarto, Córdoba.

CASSANY, D. 2003. Aproximaciones a la lectura crítica: teoría, ejemplos y reflexiones. Recuperado de: https:// repositori.upf.edu/bitstream/handle/10230/21224/Cassany_TARBIYA_32.pdf?sequence $=1$

CASTILLO, 2000. Citado por: Aguirre, D. En: Reflexiones acerca de la competencia comunicativa profesional. Facultad de Ciencias Médicas Finlay-Albarrán. La Habana, 2005

EDITA: Organización de Estados Iberoamericanos para la Educación, la Ciencia y la Cultura (OEI)

FERNÁNDEZ, G. Y CARLINO, P. (2010). ¿En qué se diferencian las prácticas de lectura y escritura de la universidad y las de la escuela secundaria?. LECTURA Y VIDA, 31 (3) 6-19.

GIRÓN, M y VALLEJO, M., (1992). Producción e interpretación textual. Medellín: Editorial Universidad de Antioquia, ICFES, (2014). Manual de Lectura Critica. En: http: / / www.icfes.gov.co/item/1640

IRAGUI, (1997-2016). Centro Virtual Cervantes (C) Instituto Cervantes. http://cvc.cervantes.es/ ensenanza/biblioteca ele/

MINISTERIO DE EDUCACIÓN NACIONAL. Ley General De Educación. Colombia. 1994.

NIÑO, 2008. Citado por: Lily Bermúdez y Liliana González, Revista Quorum Académico (2011).
OCAZIONEZ, 2007 "Las competencias transversales en el currículo de las unidades tecnológicas de santander"

PANTOJA, S. 2007. Significados de la Transversalidad en el currículum: Un estudio de caso. Revista Iberoamericana de Educación (ISSN: 1681-5653) n. ${ }^{\circ} 43 / 2$ - 10 de junio de 2007 EDITA: Organización de Estados Iberoamericanos para la Educación, la Ciencia y la Cultura (OEI) Universidad Católica de Chile.

PEÑA. L. 2008. La competencia oral y escrita en la educación superior. En:http://www.mineducacion.gov.co / 1621 / articles-189357_archivo_pdf_comunicacion.pdf

PÉREZ, M y RINCÓN, G. (2013) ¿Para qué se lee y se escribe en la universidad colombiana? Un aporte a la consolidación de la cultura académica del país. Pontificia Universidad Javeriana.

REDÓN, S. (2007) Revista Iberoamericana de Educación (ISSN: 1681-5653) n. ${ }^{\circ} 43 / 2$ - Universidad Católica de Chile. 10 de junio de 2007

Rincón Castellanos, Carlos Alberto. (2011a). La competencia comunicativa, unidad 11.

TORRES, A. FERNANDEZ E. (2014). Problemas conceptuales del curriculum. Hacia la implementación de la Transversalidad curricular. Universidad de Zulia. Venezuela.

ZERPA, C, GARCÍA, M. y DONADO, T. (2014) Comprensión del Texto Académico, formación de conceptos y enfoque investigativo. Cuaderno docente I. Universidad Autónoma del Caribe. Barranquilla.

ZERPA, C. DONADO A. (2014). Evaluación de la Comprensión Lectora. Cuaderno docente II. Universidad Autónoma del Caribe. Barranquilla. 